\title{
DISCO DE SECCHI Y CLOROFILA «a» EN DOS EMBALSES DE AGUAS MINERALIZADAS
}

\author{
G. Moyá y G. Ramón \\ Facultad de Ciencias. Universidad de Palma de Mallorca.
}

Palabras clave: Transparency. Clorophyll. Trophic state. Reservoirs.

\section{ABSTRACT \\ SECCHI DISK AND CLOROPHYLL «a» IN TWO RESERVOIRS OF MINERALIZED WATERS}

Secchi disc depths and chlorophyll «a» concentration at different levels of the water column were obtained in two Majorican reservoirs.

The monthly transparency values during two annual cycles showed a lower chlorophyll «a» contnbution to light extinction in Cúber reservoir.

Secchi disc-chlorophyll relationships were obtained and related with other general equations derived from lake and reservoir data.

\section{INTRODUCCION}

La extinción de la luz en ambientes acuáticos viene determinada por la cantidad de partículas eri suspensión, los pigmentos disueltos y las propias características del agua (Verduin, 1982). La concentración de clorofila «a» suele ser el componente menos constante tanto espacial como temporalmente, por lo que se acostumbra a considerar como un factor fundamental en la atenuación de la luz en las aguas naturales.

En oceanografía y limnología se utiliza el valor de la transparencia, medida con el disco de Secchi, como una estima de la extinción de la luz en el agua (French et al., 1982), que se ha intentado relacionar con la concentración de clorofila en el medio y ha sido utilizado como indicador de la calidad del agua (Shapiro et. al., 1975).

Las relaciones entre disco de Secchi y clorofila formuladas como generalizaciones de aplicación amplia han sido objeto de criticas y polémicas (Lorenzen, 1980; Megard et al., 1980; Edmondson, 1980; Carlson, 1980; Osgood, 1982; entre otros).

En este trabajo se presentan las relaciones encontradas para estos dos parámetros en un sistema de dos embalses en cadena, de cara a un más simplificado

Limnetica 3: 165-170 (1987)

(C) Asociación Española de Limnologia, Madrid. Spain seguimiento posterior de los mismos, y se comparan con relaciones similares establecidas a nivel general.

\section{MATERIAL Y METODOS}

Cúber y Gorg Blau constituyen un sistema de dos embalses en cadena ubicados en la porción central de la Serra de Tramuntana (Mallorca). Las aguas de los mismos poseen un elevado grado de mineralización, en concordancia con las características litológicas de la zona en que se asientan, y son utilizadas para el abastecimiento de la ciudad de Palma.

El estudio limnológico de ambos embalses, realizado durante un período de dos ciclos anuales, desde octubre de 1976 hasta octubre de 1978, en que se visitaron con una periodicidad mensual, posibilitó la obtención de una amplia información relativa a las características físicas y químicas de las aguas y del fitoplancton presente en ellas.

Los resultados de la transparencia del agua se obtuvieron haciendo uso de un disco de Sechhi (Tyler, 1968) de color blanco y de $30 \mathrm{~cm}$. de diámetro, anotando el valor promedio de las mediciones obtenidas al bajar y al subir el disco. La concentración de cloro- 


\begin{tabular}{|c|c|c|}
\hline $\begin{array}{c}\text { TRANSPARENCIA } \\
(M)\end{array}$ & $1.30^{3.64} 7.30$ & $2.50^{4.88} 8.30$ \\
\hline$\underset{\left(M G / M^{3}\right)}{C L O R O F I L A}$ & & \\
\hline EN SUPERFICIE & $0.97^{2.81} 8.57$ & $0.67^{3.22} 9.62$ \\
\hline $\begin{array}{l}\text { MEDIA, EN TODA } \\
\text { LA COLUMNA }\end{array}$ & $2.72^{3.70} 8.43$ & $2.31^{4.08} 9.92$ \\
\hline $\begin{array}{l}\text { ACUMULADA }\left(\mathrm{MG} / \mathrm{M}^{2}\right) \\
\text { A } Z_{D S}\end{array}$ & $2.72^{9.08} 18.62$ & $5.37^{15.74} 28$. \\
\hline
\end{tabular}

Tabla 1.-Valores medios, mínimos y máximos para cada uno de los parámetros considerados en los dos embalses. Average values, minimum and maximum for clorophyll and transparency in the two reservoirs.

Ds , $c^{0.68}=7.7 \quad \begin{array}{ll}\text { SHAPIRO ET AL, } \\ \text { CARLSON, } 1977\end{array} \quad$ LAGOS

Ds . $c^{0.47}=3.55$ BAKER ET AL, 1981 LAGOS DE FLORIDA

Ds . $c^{0.17}=3.5 \quad$ RuLL ET AL, $1984 \quad$ EMBALSES ESPAÑOLES

Ds . $c^{0.44}=5.58$ RULL ET AL., $1984 \quad$ EMBALSES ESPAÑoLES, PRIMAVERA

Ds,$C^{0.52}=6.67 \quad$ ORTIZ ET AL, $1983 \quad$ EMBALSES ESPAÑOLFS

Ds. $c^{0.51}=9.33 \quad$ O.C.D.E., $1982 \quad$ Síntesıs (LAgos y EMBaLses)

Tabla 2.-Relaciones entre disco de Secchi y Clorofila «a» que aparecen referidas en el texto.

Some relationship between Secchi disk transparency and Chlorophyll «a». 

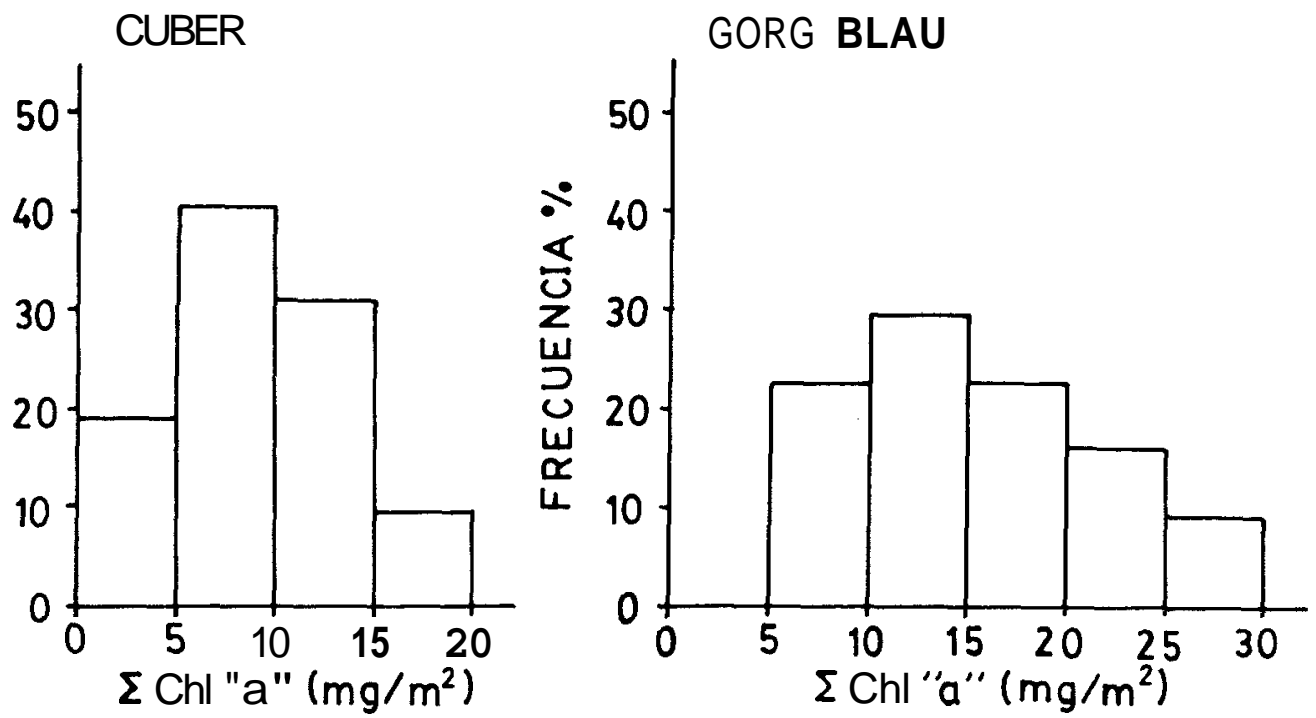

Figura 1.-Histograma de frecuenciasde los valores de la transparencia medida con el disco de Secchi, en función de la cantidad total de clorofila «a» a dicho nivel.

Histogram ofthe wole chlorophyll «a» at the Secchi disk depth.

fila «a» se determinó mediante espectrofotómetro, haciendo uso de metanol (90\%) como solvente y aplicando la ecuación de Talling y Driver (Vollenweider, 1974).

\section{RESULTADOS Y DISCUSION}

Los resultados obtenidos en los dos embalses, a lo largo del período referido y para las dos variables consideradas, se han recogido de forma sintética en la tabla 1

De entrada cabe reseñar las sensibles diferencias entre ambos embalses, a pesar de su proximidad y similitud en las características químicas de sus aguas (Moya \& Ramón, 1981).

Los valores de la transparencia, medida con el disco de Secchi, en el embalse de Cúber son inferiores en un metro a los correspondientes al embalse de Gorg Blau. No obstante, la concentración de clorofila en las aguas del embalse de Cúber es inferior, tanto en superficie como en promedio en toda la columna de agua, a la determinada en el embalse del Gorg Blau. Ello permite deducir una desigual incidencia de la concentración de clorofila en el agua sobre la profundidad de visión del disco de Secchi en ambos embalses. Su importancia es mucho mayor en el embalse del Gorg Blau; mientras en el embalse de Cúber tienen bastante importancia componentes distintos a los pigmentos y que corresponderían, mayoritariamente, a una mayor proporción de sólidos en suspensión en las aguas de este último embalse.

Un buen indicador de la influencia ejercida por la concentración de clorofila sobre el valor de la transparencia medida con el disco de Secchi viene representado por la concentración de clorofila acumulada a la profundidad a que deja de verse el disco. Los resultados para este parámetro también se han incluido en la tabla 1, y es claramente constatable la notable diferencia existente entre ambos embalses, con una media muy inferior para el embalse de Cúber. Además la oscilación entre valores extremos es muy superior en el embalse del Gorg Blau, cuando el rango de la misma es similar en ambos embalses.

En la figura 1 se ha representado la distribución en clases de la cantidad de clorofila «a» acumulada por unidad de superficie a la profundidad de visión del disco de Secchi en ambos embalses. La primera clase considerada no ha tenido incidencia alguna en el embalse del Gorg Blau, mientras incluye prácticamente una quinta parte de las mediciones efectuadas en el embalse de Cúber. Igualmente notoria es la diferencia cuando se comparan las dos primeras clases consideradas. Asimismo cabe considerar la importancia de las clases más altas para el embalse del Gorg Blau, con unos mínimos de transparencia superiores a los del embalse de Cúber. Todo ello redunda en el hecho apuntado de una mayor incidencia de la clorofila en la atenuación de la luz en el embalse del Gorg Blau; mientras en el Cuber es considerable la importancia 

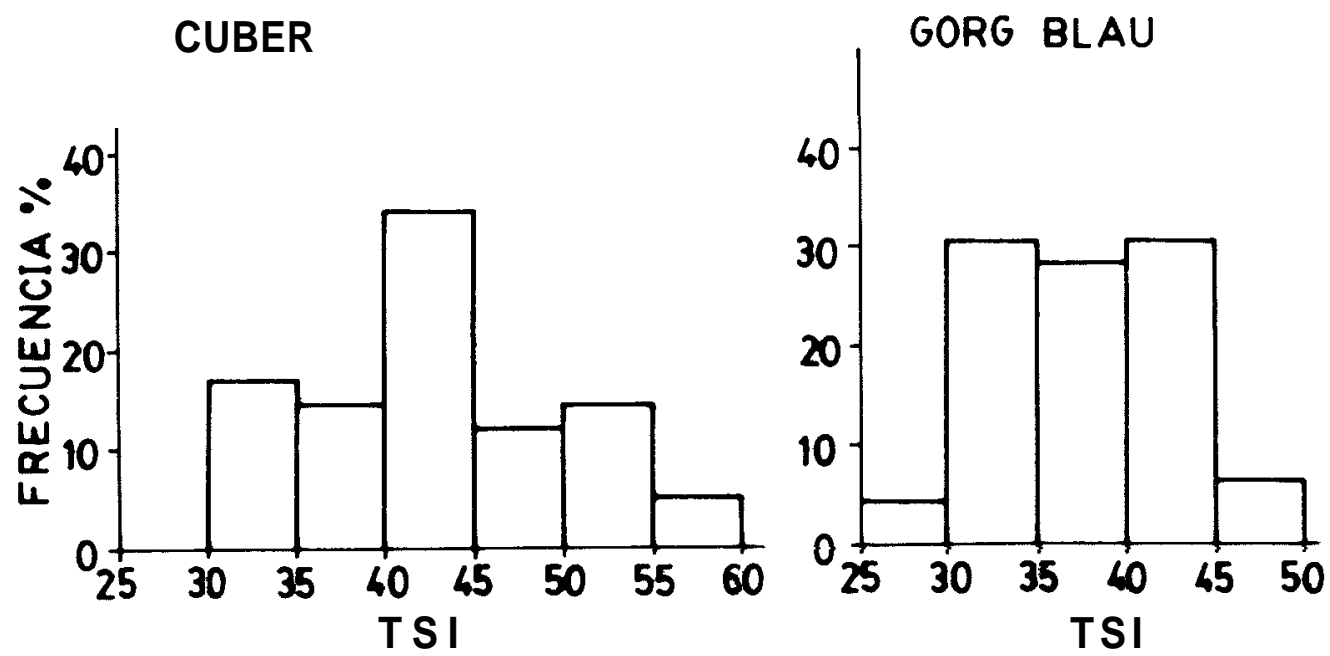

Figura 2.-Relación entre los logaritmos de la profundidadde visión del disco de Secchi y la concentración de clorofila en superficie en los embalses de Cúber (izquierda)y Gorg Blau (derecha).(U mezcla, O estratifición).

Secchi disk-chlorophyll relationships in Cúber (left)and Gorg Blau (nght)reservoirs. (O mixing, O stratification).

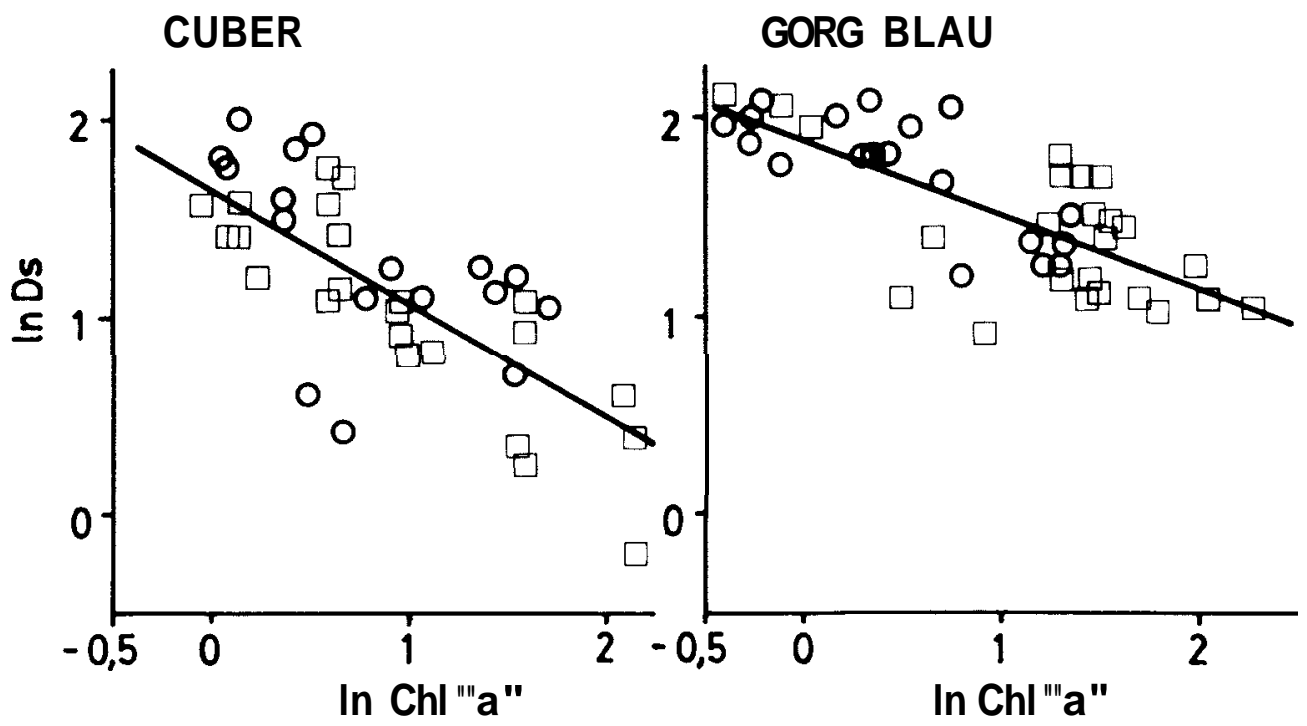

Figura 3.-Histograma de frecuencias de los valores del índice del estado trófico (TSI)en los embalses de Cúber (izquierda)y Gorg Blau (derecha).

Histogram of the Trophic State Index (TSI)values in Cúber (left)and Gorg Blau (right) reservoirs.

detentada por las partículas en suspensión. No obstante, las diferencias apuntadas también pueden haberse visto condicionadas en parte por la distinta morfometría de ambos embalses (Ramón y Moya, 1982).

La mayor o menor participación de los pigmentos en la atenuación de la luz no indica sin embargo el grado de correlación existente entre ambos parámetros en uno y otro embalse. A tal efecto se ha procedido al cálculo de la recta de regresión entre ambos parámetros, para la clorofila se ha considerado tanto la concentración en el nivel de agua superficial como la cantidad total acumulada por unidad de superficie a la profundidad de visión del disco de Secchi. Los re- 
sultados alcanzados en uno y otro caso son muy similares y a tal efecto se ha optado por representar la relación existente con respecto al valor de clorofila en la superficie del agua (figura 2) dada su mayor simplicidad y posible utilización futura para estos dos sistemas concretos.

En ambos embalses el ajuste de los valores encontrados es bastante bueno y con unos coeficientes de correlación claramente significativos $(r=-0,67$ y $r=$ $-0,75$ ), si bien, como cabía esperar, sensiblemente mejor en el caso del embalse del Gorg Blau. A destacar las mínimas diferencias, prácticamente nulas, cuando se consideran la totalidad de valores obtenidos en uno y otro embalse (fig. 2), al que separar los valores correspondientes a mezcla y estratificación (figura 2), y al separar las estaciones del embalse en las que se han obtenido.

Los datos hallados permiten establecer una expresión que define la relación entre el valor de la transparencia medida con el disco de Secchi y expresada en metros $\left(D_{s}\right)$ y la concentración de clorofila, expresada en $\mathrm{mg} / \mathrm{m}$. ${ }^{3}$, en la capa de agua superficial (C) en uno y otro embalse. Estas expresiones resultan ser:

$$
\begin{aligned}
& D_{\mathrm{s}} \cdot \mathrm{C} 0,75=5,26 \text { (para el embalse de Cúber) } \\
& \mathrm{D}_{\mathrm{s}} \cdot \mathrm{C} 0,36=6,49 \text { (para el embalse del Gorg } \\
& \text { Blau) }
\end{aligned}
$$

Las diferencias entre una y otra son evidentes pese a la similitud en las características de las aguas y la conexión existente entre uno y otro embalse. Estas expresiones también difieren de la referida por Shapiro y colaboradores (1975) obtenida con un tratamiento idéntico al nuestro a partir de valores procedentes de lagos y que ha sido aplicada en un amplio grupo de embalses españols (Margalef, et al., 1976). Igualmente se observan diferencias, en un sentido u otro, respecto a otras expresiones halladas mediante tratamiento más o menos distinto de los valores encontrados en lagos americanos (Baker et al., 1981) o en embalses peninsulares (Ortiz et al., 1983; Rull et al., 1984) y lo mismo puede decirse respecto a las expresiones obtenidas en informes de síntesis (OCDE, 1982), que aparecen recogidas en la tabla 2.

Todo ello redunda en el interés que posee el conocimiento de tales expresiones para un sistema concreto, ya que las generalizaciones no pueden ser aplicadas con una cierta garantía en todos los casos y menos aún cuando se trata de embalses.

La formulación de un índice del estado trófico (TSI) de las aguas en función de los valores de la transparencia, medida con el disco de Secchi y expresada en metros (Shapiro et al, 1975; Carlson, 1977), y el hecho de que dicho índice fuera aplicado a un amplio conjunto de embalses peninsulares (Margalef et al., 1976) nos ha movido a aplicarlo también a los dos embalses considerados. Los resultados obtenidos se han sintetizado en la figura 3.

Las diferencias entre uno y otro embalse son claras. En el embalse de Cúber se observa una concentración de los valores hallados en torno a uno perfectamente equiparable al encontrado de modo mayoritario en los embalses peninsulares (40-50), y acompañado de una dispersión más o menos amplia. En el embalse del Gorg Blau no aparece una clase claramente preponderante y además los valores obtenidos son algo inferiores a la media hallada en los embalses peninsulares.

Estas diferencias entre los dos embalses respecto a los valores para el índice del estado trófico pueden ser explicadas por la mayor incidencia de las partículas en suspensión en las aguas del embalse de Cúber, lo que lo hace más similar a la mayoría de los embalses; mientras que el embalse del Gorg Blau posee unas características particulares que, en múltiples ocasiones, lo hacen más similar a un lago que a un embalse típico.

\section{BIBLIOGRAFIA}

Baker, L. A.; P. L. Brezonik \& Ch. R. Kratzer. 1981. Nutrient louding-trophic state relationships in Florida lakes. Water Resources, research center. Univ. Florida. Publication n. ${ }^{\circ} 56.126$ págs.

Carlson, R. E. 1977. A trophic state index for lakes. Limnol. Oceanogr., 22: 361-369.

Carlson, R.E. 1980. More complications in the chlorophyllSecchi disk relationship. Limnol. Oceanogr., 25: 379-382.

Edmondson, W. T. 1980. Secchi disk and chlorophyll. Limnol. Oceanogr., 25: 378-379.

French, R. H.; J. J. Cooper \& S. Vigg. 1982. Secchi disc relationships. Water Res. Bull., 18: 121-123.

Lorenzen, M. W. 1980. Use of chlorophyll-Secchi disk relationships. Limnol. Oceanogr., 25: 371-372.

Margalef, R.; D. Planas, J. Armengol, A. Vidal, N. Prat, A. Guiset, J. Toja \& M. Estrada. 1976. Limnología de los embalses españoles. Dirección General de Obras Hidráulicas. Ministerio de Obras Públicas. Madrid. 2 vols. $422+85$ págs.

Megard, R. O.; J. C. Settles, H. A. Boyer \& W. S. Combs Jr. 1980. Light, Secchi disks, and trophic states. Limnol. Oceanogr. 25: 373-377.

Moyá, G. \& G. Ramón. 1981. Contribución al conocimiento de la mineralización de las aguas de los embalses de Cúber y Gorg Blau y de sus principales aportes. Boll. Soc. Hist. Nat. Balears, 25: 21-30.

OCDE. 1982. Eutrophisation des euux. Méthodes de survrillance, d'évaluation et de lutte. OCDE. Paris. 164 págs.

Ortiz, J. L.; R. Peña, G. F. Lee \& R. A. Jones. 1983. Aportación de nutrientes y eutrofización de embalses. Centro de Es- 
tudios Hidrográficos. Ministerio de Obras Públicas y Urbanismo. Madrid.529 págs.

Osgood, R. A. 1982. Using diferences among Carlson's trophic state index values in regional water quality assessment. Water Resources Bull., 18: 67-74.

Ramón, C. \& C. Moyá. 1982. Caractensticas morfológicas y morfométricas de los embalses de Cúber y Gorg Blau (Mallorca). Boll. Soc. Hist. Nat. Balears, 26: 145-150.

Rull, V.; T. Vegas \& J. Navarro. 1984. Extinción de la luz en los embalses españoles. Relaciones con la concentración de clorofila y las partículas en suspensión. Oecologia aquatica, 7: 25-36.
Shapiro, J.; J. B. Lundquist \& R. E. Carlson. 1975. Involving the public in limnology - an approach to communication. Verh. Internat. Verein. Limnol., 19: 866-874.

Tyler, J. E. 1968. The Secchi disc. Limnol. Oceanogr., 13: $1-6$.

Verduin, J. 1982. Components contributing to light extinction in natural waters: Method of isolation. Archiv. Hydrobiol., 93: 303-312.

Vollenweider, R. A. (ed.). 1974. A Manual on Methods for Measuring Prirnary Production in Aquatic Environments. IBP Handbook N. ${ }^{\circ}$ 12. Blackwell Scientific Publications. Oxford. 2nd ed. 225 págs. 